\title{
A Framework for Resource Allocation Strategies in Cloud Computing Environment
}

\author{
Muhammad Asad Arfeen, Krzysztof Pawlikowski, Andreas Willig \\ Department of Computer Science \& Software Engineering \\ University of Canterbury \\ Christchurch, New Zealand. \\ muhammad.arfeen@pg.canterbury.ac.nz \\ \{krys.pawlikowski, andreas.willig\}@ canterbury.ac.nz
}

\begin{abstract}
This paper presents a critical evaluation of current resource allocation strategies and their possible applicability in Cloud Computing Environment which is expected to gain a prominent profile in the Future Internet. This research attempts to focus towards network awareness and consistent optimization of resource allocation strategies and identifies the issues which need further investigation by the research community. A framework for resource allocation in Cloud Computing, based on tailored active measurements, has also been proposed. The main conclusion is that network topology, traffic considerations, changing optimality criteria along with dynamic user requirements will play a dominant role in determining future Internet application architectures and protocols, shaping resource allocation strategies in Cloud Computing, for example.
\end{abstract}

Keywords- Cloud Computing, Distributed Systems, Resource Allocation, Optimization.

\section{INTRODUCTION}

There has been a lot of research work on the analysis of networks, and the Internet in particular, from the point of view of data traffic and security management and various resource allocation algorithms have been proposed [1-5]. Given the fact that resources on the Internet have been always limited and traffic is going to increase unbounded, there is a growing opinion that performance of Internet architectures should be evaluated online, so as to make Internet more reactive and responsive to new services like cloud computing which will face more traffic intensity and delay sensitiveness in near future. There is a need for developing new methods of reliable active measurements aimed at capturing global Internet behavior online, and for enabling prediction of the most critical performance parameters. Reliable and predictable metrics and the methodologies of their online collection and analysis, as mentioned in [14,17-18], should be used to fine tune or optimize existing and future global Internet applications. Besides measuring network performance, the probing can also be used at the system or middleware level, for assessing the performance of disks, processors, memories and other components of such cooperative environments as grid or cloud computing systems [9]. So adjusting the granularity level of active probes is also an important research task, with solutions which could depend upon the current network situation and user demands.

Based on topological changes, routing asymmetries and packet losses, it is also possible that active measurements may give misleading results about current network conditions[15-16], so online identification of inaccurate measurements is necessary as well. If a wrong online control decision, e.g. related with allocation of a resource, was made on the basis of inaccurate measurements, then the associated cost or overhead of the corresponding impact should be quantified, to assess its possible cost and to allow its later adjustment, e.g. reallocation of resources. More importantly, the limits on the accuracy of active measurements [10] need to be investigated so that they can be credible.

Furthermore, there is a need of extending the existing analytical models, to use them in studies of performance of new Internet services [6-8]. This will definitely help in investigating performance unpredictability problems in larger network environments, such as global Internet and such its future applications as Cloud Computing. Performance models, which are able to properly quantify user's (changing) requirements into decision variables, need to be investigated and assessed by online measurements and monitoring. Efforts should be made towards making performance models predictive and responsive to simultaneous changes of multiple decision variables.

Such research activities require appropriate tools. Fortunately, these tools have become widely available with development of global experimental networking facilities such as PlanetLab[13]. With their help one can experiment and assess feasibility of applications of new measurement methodologies, resource allocation strategies and their implementations in full scale cooperative networking environments. 


\section{A CRITICAL EVALUATION OF CURRENT RESOURCE ALLOCATION STRATEGIES}

Previous efforts aimed at developing new Internet services, such as e.g. Utility Computing, have been unable to gain significant adoption by industry because they lack at least one of the following important characteristics, identified as essential for Cloud Computing and other future Internet's environments, [21] :

a. on demand availability of seemingly infinite computing resources;

b. complete technical and economical flexibility in the use of hardware resources according to organization's requirements, and

c. short term acquisition and release of resources.

In addition, lack of proper measurement, monitoring and online control infrastructures have rendered many efforts on the Future Internet's resource allocation algorithms unable to adapt to network dynamics[11-12]. Moreover the dynamics of the Internet is expected to escalate and prediction of its performance will be more difficult in future. It is highly probable that any effort towards a new service development and optimization, without considering the related performance issues, will have no chance to gain significant applicability and adoption by industry.

Pervasive supply of seamless and transparent access to heterogeneous resources and services, such as network domains, applications, services and storage owned by multiple organizations, pose many challenges for measuring, controlling and balancing resource access [24]. It is important for a resource reservation strategy not to be static as changing network dynamics and users' requirements may lead a seemingly optimal allocation to an under-provisioned or over-provisioned state at a later time instant. The allocation strategy should be adaptive to the changes in resource management policies and the current network traffic situation. Additionally, the allocation strategy needs to cope with users' and administrators' optimality criteria which are usually opposite and may hinder a given allocation strategy to converge towards an optimal allocation and equilibrium.

Resource allocation algorithms employ centralized or decentralized strategies, deriving concepts from artificial intelligence, theory of random graphs and peer-to-peer based systems. Below is a brief critical survey of these strategies.

\section{Artificial Intelligence (AI) based approaches}

The problem of network resource allocation in Cloud Computing has been considered from two major perspectives: game theory and, heuristic methods like Particle Swarm Optimization and Genetic Algorithms.

Applications of Game theory strategies for resource allocation, bandwidth sharing and pricing models have been demonstrated in $[2,25]$. The ultimate goal of the game-theoretic methods is to maintain Nash Equilibrium state among competing users of cloud services. Nash Equilibrium is a concept of game theory where the optimal outcome of a game is one where no player has an incentive to deviate from his or her chosen strategy after considering opponent's choices. Overall, an individual can receive no incremental benefit from changing actions, assuming other players remain constant in their strategies. A game may have multiple Nash equilibria or none at all. It is implemented in two phases[2]. In first phase, each individual competitor solves the optimal schedule using binary integer programming (a type of linear programming method targeted to maximize individual profit or minimize loss) without consideration of multiplexing of resource assignments. In the second phase, using an evolutionary mechanism, changes are made in the initial optimal solutions of individual competitors, to have overall or global multiplexed strategies with minimizing individual's required efficiency losses. The proposed strategy is valid in case of independent and dependent tasks scheduling. Dependent task scheduling is considered as an NP-hard problem [2, 26]. The major emphasis in this approach is on the communication between dependent tasks. The current performance of underlying network and changing user requirements are not considered. Also complete specification of dependency and level of communication among dependent subtasks is needed in advance, which may not be possible in many cases of scientific and business computations.

Particle swarm optimization (PSO) is a population based stochastic optimization technique inspired by social behaviors, like bird flocking or fish schooling. PSO shares many similarities with Genetic Algorithms (GA) but there are fewer parameters to adjust as compared to GA [27] . Based on test cases, the proponents of this approach have concluded that performance of PSO-based scheduling is better than Genetic Algorithm based approaches and requires fewer controlling parameter as it correlates particle's position to task-resource mappings [28]. The goal here is to find a task-resource mapping such that total highest cost among all compute resources is minimized. Tasks are considered as particles and their dimension is the number of tasks in the workflow. For PSO-based scheduling it is necessary to know average computation 
times of tasks in advance, which may not be possible in many cases. Additionally, a PSO-based algorithm [28] uses current network load to update average communication cost between resources but it does not attempt to reallocate/reschedule tasks if better network conditions are available at other times. An important research issue is live migration/rescheduling of tasks to a better network or in case of some fault or losses in existing network which is serving the tasks.

It is noteworthy that the research in the area of Genetic Algorithms [29-31] has identified that, although approaches based on heuristics like PSO require less configuration parameters, they may not lead to the optimal solution. However, it is also noteworthy that, for an online decision on optimal resource scheduling, the performance analysis of GA-based approaches needs further research.

\section{Approaches based on theory of random graphs}

Random graphs provide an important paradigm which may be used to investigate the connectivity of a network and, more specifically, the optimal vertex distribution in a resource network. Application of stochastic processes like Random Walks on random graphs has been recently investigated in [24] for achieving a load balancing objective. A decentralized scheme has been considered which generates regular resource allocation networks, so as to achieve distributed and moreover a cooperative load balancing [24]. The specific feature of this cooperative random walk based strategy is that heavily loaded nodes expose their current situation to an incoming task which carries current load information of all other nodes, which it encounters during the walk. The maximum random walk length, in terms of the number of hops, is limited and the last node can determine the least loaded node and forward the task to that node [24]. The strategy can be regarded as self-configuring and self-optimizing. The main inspiration came from Erdos-Renyi (ER) random graphs [32], using the mapping between connectivity of a resource node and its current load. In ER random graphs the probability of deviation from average connectivity decreases exponentially with the deviation distance. It is ensured that connectivity distribution of the resource network corresponds to a random graph, thereby balancing the load distribution equitably across all nodes with computing resources. This work also introduces Biased Random Sampling (BRS), by using biased random walk which is a policy based sampling strategy to allocate resources or determine the pattern of the random walk, e.g. if considering geographical distance in random walk or any other user generated utility measure. Both network environments with homogeneous nodes (of the same computing capacity) and heterogeneous nodes (of different computing capacity) are considered.
The length of random walk is an important performance criterion but no formulated mechanism has been presented so far, except static bounds on the hop count (the length of random walk). The problem of allocation of dependent tasks, i.e. scheduling a workflow represented in a dependent task system using a directed acyclic graph, needs further investigation as well.

\section{Peer-to-Peer based approaches}

Inter-domain scheduling of grid applications is an important research task due to difficult provisioning of the same QoS requirements and strict threads/processes concurrency requirements across multiple domains. Frameworks like General-purpose Architecture for Reservation and Allocation (GARA) [33] , Network Resource Scheduling Entity (NRSE) [34], and Grid Quality of Service Management (G-QoSM) [35], pay more attention towards the available computing capacity of resources, thus it may be possible that a lightly loaded node may get selected in a highly congested network, and this will degrade overall user's experience if the job requires high network I/O [3]. P2P-based approaches address resource allocation problem in a single administrative domain by using a typical broker, like Grid Network Resource Broker (GNB) [36] which is composed of job scheduler, resource monitor and bandwidth broker.. The bandwidth broker requires a direct access to routers but it cannot administer all routers, so there exists a scaling problem associated with this technique, though it works well if the bandwidth broker is overall in-charge of the administrative domain. The resource monitor needs to use the same information in all the domains even if the computing resources belong to different administrative domains. The P2P-based techniques use a specific type of Routing Index (RI), called Hop-Count Routing Index (HRI) [37], which represents a specific type of neighboring computing resource. HRI takes into account the number of hops, aggregate quality of neighbors, their processing power, current load and effective bandwidth. It is important to maintain a consistent HRI which is the core of P2P-based allocation strategies but this consistency of HRI may not be possible across multiple domains. GridSim [38] has been used in simulation studies of this technique but it will be worthwhile to use experimental testbeds built e.g. on PlanetLab[13] to have more credible and valid results from its full scale deployment. It is also noteworthy that P2P overlay routing may intensify congestion at the Internet's backbone because current traffic engineering algorithms implemented by Internet Service Providers (ISPs) are oblivious to P2P traffic demands [39]. 


\section{PROPOSED FRAMEWORK FOR RESOURCE ALLOCATION IN CLOUD COMPUTING}

In this Section we present a new research framework for the resource allocation problem. The practical directives are briefly presented first, and it is followed by the problem's mathematical formulation.

\section{- Tailored Active Measurements for Resource Allocation in Cloud Computing}

The problem of resource allocation in a highly distributed and dynamic environment can be considered in a new way, based on online Internet measurements. As a prototype, the following types of elastic (extendable) network resources can be considered for allocation:

\section{Computing resources}

2. Storage resources

The Global Cloud Monitor \& Controller will instantiate and monitor Local Cloud Front Ends. The Local Cloud Front-End, having received user's workflows (usually represented by directed acyclic graphs), will quantify the required performance metrics of each particular workflow and send tailored active probes accordingly, to find the optimal resources in terms of the required performance metrics and the current network situation. Tailoring of active probes will be attempted in such a way that the decided allocation of resources becomes a true reflection of the current user requirements, while remaining sensitive to possible changes (expectedly increase) in the user's requirements in the nearest future. It can then schedule the workflow monitoring the Service Level Agreements [19]. A conceptual diagram of this framework is presented in Figure 1.

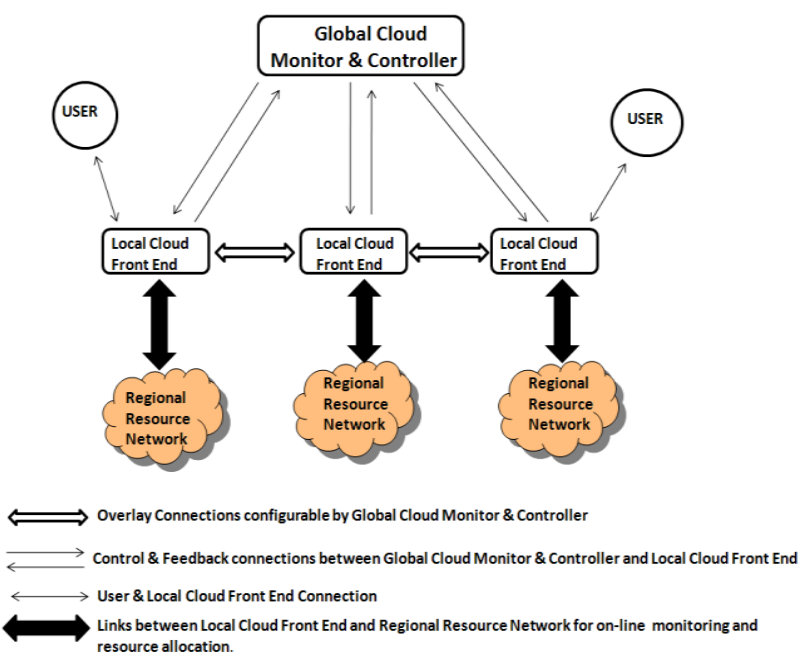

Figure 1: Conceptual diagram of proposed framework
In order to have more reliable and valid results, it is suggested that proposed framework should be tested over global Internet experimental testbeds, such as PlanetLab [13]. It is expected that if researchers adopt this framework then it may result in more efficient, scalable and fault-tolerant execution environment for parallel programming environments such as Google's MapReduce [22] and the open-source version of it, i.e. Apache's Hadoop [23].

\section{- Optimization of Network Resource Allocation}

Due to stochastic nature of networks, it is likely that allocation of resources at a particular time (on the basis of active measurements and user-level performance metrics) may not remain optimal even over a relatively short period of time. It is also possible that, due to elastic nature of cloud resources, more resources may be added/removed later on. This can render current allocation not an optimal one. Thus, the optimization is necessary to cope with:

1. Non-stationarities in Internet traffic;

2. Dynamic changes in network topology or Routing ;

3. Dynamic changes in resource connectivity or the associated costs ; and

4. Changing user requirements (online \& offline)

As this is a dynamic resource allocation problem, so it will be worthwhile to investigate the applicability of such methods as, for example, approximate dynamic programming techniques [20]. Scheduling-decision making over time under uncertainty can be investigated both from finite and infinite time horizon perspective. The latter will give further insight into long term behavior and effects of decisions. As the first step towards achieving this goal, a specific model of resource allocation related to Cloud Computing can be derived from the General Resource Allocation Problem [20]. This multidimensional resource allocation problem class suffers from all the three curses of dimensionality [20], namely uncertainties in state space, outcome space and the action space. Therefore optimization of resource allocation strategy based on online Internet measurements can be a interesting and challenging task.

A classical optimal resource allocation problem can be presented as:

$$
\begin{array}{ll}
\text { minimize or maximize } & f\left(x 1, x 2 \ldots \ldots, x_{n}\right) \\
\text { subject to } & \sum_{j=1}^{n} x_{j}=N \\
\text { with } & x_{j} \geq 0, \quad j=1,2 \ldots . . n
\end{array}
$$


where $N$ is the total number of resources to be allocated to $n$ activities, so that objective value function $f\left(x 1, x 2 \ldots \ldots, x_{n}\right)$ becomes minimized (e.g. cost or loss) or maximized (e.g. profit). The variable $x_{j}$ represents the amount of resources associated with activity $j$. In the case of Cloud Computing, the resources are elastic, i.e. $N$ cannot be regarded as static parameter. Therefore, it is an important research task to investigate optimal resource allocation algorithms, as presented in [40], but not assuming $N$ as a fixed value. Thus online monitoring of $N$ is necessary to decide the best time for allocation or reallocation towards optimization.

\section{CONCLUSION}

On the basis of this critical review of existing strategies of resource allocation in Grid/Cloud computing environment one can conclude that lack of proper measurement, monitoring and online control infrastructure has rendered many efforts on Future Internet resource allocation algorithms unable to adapt to dynamics of Internet. Moreover the dynamics of Internet has rendered traffic prediction and capacity planning more difficult due to increase in various kinds of nonstationarities in Internet traffic and user behaviors. The evaluation of existing resource allocation strategies highlights the need for further investigating performance unpredictability problems in a larger network environment like global Internet and its future applications, such as, Cloud Computing. Performance models, which are able to properly quantify user's (changing) requirements into decision variables, are needed to be investigated and assessed by online measurements and monitoring towards optimization. More research efforts are needed to make these performance models predictive and responsive to simultaneous changes of multiple decision variables. The proposed framework based on tailored active measurements for optimal resource allocation is currently basis of our future research activities.

\section{ACKNOWLEDGMENT}

This research study and future implementation of the proposed framework has been partially supported by PlanetLab New Zealand under Research Education And Networks New Zealand (REANNZ).

\section{REFERENCES}

[1] J Choi, KS Yu, J Park, Y Mun, "Design of Network Aware Resource Allocation System for Grid Applications," in Computational Science and Its Applications - ICCSA 2006. vol. 3981, ed: Springer Berlin / Heidelberg, 2006, pp. 525-533

[2] G. Wei, AV Vasilakos, Y Zheng, N Xiong ,"A gametheoretic method of fair resource allocation for cloud computing services," The Journal of Supercomputing, vol. 54, pp. 252-269, 2010.
[3] A Caminero, O Rana, B Caminero, "Improving Grid Inter-Domain Scheduling with P2P Techniques: A Performance Evaluation," in Grid and Cooperative Computing, 2008. GCC '08. Seventh International Conference, 2008, pp. 190-200.

[4] S. Alexander and S. Thomas, "Network-aware migration control and scheduling of differentiated virtual machine workloads," presented at the Proceedings of the 2009 ICSE Workshop on Software Engineering Challenges of Cloud Computing, 2009.

[5] C. Robert and C. Mark, "Dynamic Server Selection using Bandwidth Probing in Wide-Area Networks," Boston University1996.

[6] C. Allison, S. Purdie, A. Miller., "Responsiveness on the Interactive Grid," 2007. ICC '07. IEEE International Conference on Communications , 2007, pp. 371-376.

[7] A Ruddle, C Allison, P Lindsay, "Analysing the latency of WWW applications", Proceedings of Tenth International Conference on Computer Communications and Networks, 2001, pp. 116121.

[8] C Allison, M Bramley, J Serrano,"The world wide wait: where does the time go?," in Euromicro Conference, 1998. Proceedings. 24th, 1998, pp. 932-938 vol.2.

[9] M. Natu and A. S. Sethi, "Active Probing Approach for Fault Localization in Computer Networks," 4th IEEE/IFIP Workshop on End-toEnd Monitoring Techniques and Services, 2006, pp. 25-33.

[10] R. Matthew, "Fundamental bounds on the accuracy of network performance measurements," presented at the Proceedings of the 2005 ACM SIGMETRICS international conference on Measurement and modeling of computer systems, Banff, Alberta, Canada, 2005.

[11] T Ye, D Harrison, B Mo, B Sikdar ,"Traffic management and network control using collaborative on-line simulation,". ICC 2001. IEEE International Conference on Communications, 2001, pp. 204-209 vol.1.

[12] B Szymanski, Y Liu, A Sastry, K Madnani ,"Real-time on-line network simulation," in Distributed Simulation and Real-Time Applications, Fifth IEEE International Workshop on, DS-RT 2001., 2001, pp. 22-29.

[13] PlanetLab. Available: http://www.planetlab.org/ (accessed on 11 October 2010).

[14] M. J. P.Box, C.Reinsel, "Time Series Analysis: Forecasting Control", 4th ed.: Wiley, 2008.

[15] P. Rossi, A. Petropulu,J. Yu, F.Palmieri, G. Iannello, "Internet loss-delay modeling by use of input/output hidden Markov models", IEEE 6th Workshop on Multimedia Signal Processing, 2004, pp. 470-473.

[16] P. S. Rossi, G. Romano, F. Palmieri, and G. Iannello, "Joint end-to-end loss-delay hidden Markov model for periodic UDP traffic 
over the Internet,", IEEE Transactions on Signal Processing, vol. 54, pp. 530-541, 2006.

[17] E. D. Kolaczyk, "Statistical Analysis of Network Data : Methods and Models," in Springer Series in Statistics, ed: Springer, 2009, pp. 308-310.

[18] N. Markovich, Nonparametric Analysis of Univariate Heavy-Tailed Data: Research and Practice: Wiley, 2007.

[19] E. Marilly, O. Martinot, H. Papini, D. Goderis, "Service level agreements: a main challenge for next generation networks", ECUMN 2002. 2nd European Conference on Universal Multiservice Networks, 2002, pp. 297-304.

[20] W. B. Powell, "Approximate Dynamic Programming : Solving the Curses of Dimensionality", Wiley Series in Probability and Statistics 2007.

[21] A. F. Michael Armbrust, Rean Griffith, Anthony D. Joseph, Randy Katz, and G. L. Andy Konwinski, David Patterson, Ariel Rabkin, Ion Stoica, and Matei Zaharia, "Above the Clouds: A Berkeley View of Cloud Computing," February 10, 2009.

[22] Google's MapReduce. Available: http://labs.google.com/papers/mapreduce.html (accessed on 11 October 2010)

[23] Apache Hadoop. Available: http://hadoop.apache.org/ (accessed on 11 October 2010)

[24] M. Randles, O. Abu-Rahmeh, P. Johnson, A. Taleb-Bendiab, "Biased random walks on resource network graphs for load balancing," The Journal of Supercomputing, vol. 53, pp. 138-162, 2010.

[25] H. Yaïche, R. Mazumdar, C. Rosenberg "A game theoretic framework for bandwidth allocation and pricing in broadband networks," IEEE/ACM Transactions on Networking., vol. 8, pp. 667-678, 2000.

[26] "Approximation Algorithms for NP-Hard Problems," SIGACT News, vol. 28, pp. 40-52, 1997.

[27] PSO Tutorial. Available: http://www.swarmintelligence.org/tutorials.php (accessed on 11 October 2010)

[28] S. Pandey, L. Wu, SM. Guru, R. Buyya "A Particle Swarm Optimization-Based Heuristic for Scheduling Workflow Applications in Cloud Computing Environments,", 24th IEEE International Conference on Advanced Information Networking and Applications, 2010, pp. 400-407.

[29] H. Tarek, "Optimization of Resource Allocation and Leveling Using Genetic Algorithms," Journal of Construction Engineering and Management, vol. 125, pp. 167-175, 1999.

[30] M.R. Sherif, I.W. Habib, M Naghshineh, "An adaptive resource allocation and call admission control scheme for wireless ATM using genetic algorithms," in Global Telecommunications
Conference, 1999. GLOBECOM '99, 1999, pp. 1500-1504 vol.2.

[31] S. Palaniappan, S. Zein-Sabatto, A. Sekmen, "Dynamic multiobjective optimization of war resource allocation using adaptive genetic algorithms," in SoutheastCon 2001. Proceedings. IEEE, 2001, pp. 160-165.

[32] R. A. Erdos , On the evolution of random graphs. Publ Math Inst Hung Aczd Sci 5:17-61.

[33] A. Roy., "End-to-End Quality of Service for High-End Applications.," PhD Thesis, Dept. of Computer Science, University of Chicago, 2001.

[34] S. N. Bhatti,S. Sørensen,P. Clark,J. Crowcroft "Network QoS for Grid Systems," International Journal of High Performance Computing Applications, pp. 219-236, August 1, 2003.

[35] R. AL-Ali, S. Sohail, O. Rana, A. Hfid, G. Von Laszewsli, D.Walker, "Network QoS Provision for Distributed Grid Applications.", Intl. Journal of Simulations Systems, Science and Technology, Special Issue on Grid Performance and Dependability, vol. 5, December 2004.

[36] A. Caminero, C. Carrion and B. Caminero, "Designing an entity to provide network QoS in a Grid system," presented at the In Proc. of the 1st Iberian Grid Infrastructure Conference (IberGrid), Santiago de Compostela, Spain, 2007.

[37] A. Crespo and H. Garcia-Molina, "Routing indices for peer-to-peer systems", Proceedings of 22nd International Conference on Distributed Computing Systems, 2002, pp. 23-32.

[38] GridSim: A Grid Simulation Toolkit for Resource Modelling and Application Scheduling for Parallel and Distributed Computing. Available: $\quad$ http://www.buyya.com/gridsim/ (accessed on 11 October 2010)

[39] Hao Chen ,Zhian Gu, Dejian Ye, "Pharos: An ISP-P2P Cooperation Network Resource Allocation Architecture",CNMT 2009. International Symposium on Computer Network and Multimedia Technology, 2009, pp. 1-6.

[40] T. Ibaraki, N. Katoh, "Resource Allocation Problems: Algorithmic Approaches", Foundation of Computing series, the MIT Press, 1988. 